\title{
Regression Technique for Electromagnetic Sampling and Field Reconstruction
}

\author{
S. Rondineau ${ }^{*}(1)$, B. Fuchs ${ }^{(2)}$, F. Batista Ribeiro $\operatorname{Costa}_{(3)}^{(1)}$, L. Le Coq ${ }^{(2)}$, E. Gonçalves Sousa ${ }^{(1)}$, M.C. Migliore
}

(1) Univ. of Brasilia, Brazil, email: sebastien@unb.br, filipebatistaeng@gmail.com, eng.eduardo.gs@gmail.com

(2) IETR, Univ. of Rennes 1, France; email: benjamin.fuchs@univ-rennes1.fr, laurent.le-coq@univ-rennes1.fr

(3) DIEI, Univ. of Cassino e Lazio Meridionale, Italy; email: mdmiglio@unicas.it

The presented work deals with an alternative technique for sampling and reconstructing the electromagnetic field radiated by any kind of antenna or equivalent currents, and measured in its far field region. Based on the electrical size of the radiating object, it truncates the vector spherical modal field expansion series. Then, each component of the field is projected on the classical Fourier space for the polar dependency. This is carried out by minimizing the variance of the residual noise, or, in other words, applying the Tikhonov-Phillips regularization. This results is not only a numerically well-posed problem, but also the statistical independence of the resulting coefficients as their co-variance matrix is diagonal. Afterwards, the azimuth dependency is projected on the real valued Gegenbauer, a.k.a. ultra-spherical, polynomial family, once again following the Tikhonov-Phillips regularization scheme. It not only results in a numerically well-posed problem, but also the statistical independence of the obtained spherical modal coefficients. Moreover this double regression technique leads to the smallest two-dimensional Cartesian grid of angular sampling positions, a very useful result for the far field antenna characterization industry where measurement time has to be reduced to its minimum. Additionally, both optimum estimators and stable regularizer are extracted. Then, a statistical analysis of the residual error is performed by extracting and analyzing the noise properties and creating a statistical filter that rejects any mode that is not statistically significant through the definition of a modal signal-to-noise ratio. This result turns out to be very useful when this technique is applied in a compressive-sensing-like radiated far field antenna analysis. This technique is then applied to several different antenna measurements, as shown on fig. 1, where can be noticed a very good matching between in the far $E_{\theta}$ and $E_{\varphi}$ field 2D map in amplitude comparison of both the measured electric field and the reconstructed one for a flat metallic Luneburg lens antenna designed at $12 \mathrm{GHz}$ in the frame of MERLIN, a joint laboratory of Thalès Alenia Space and IETR. Details about this radiating structure are available in [1].

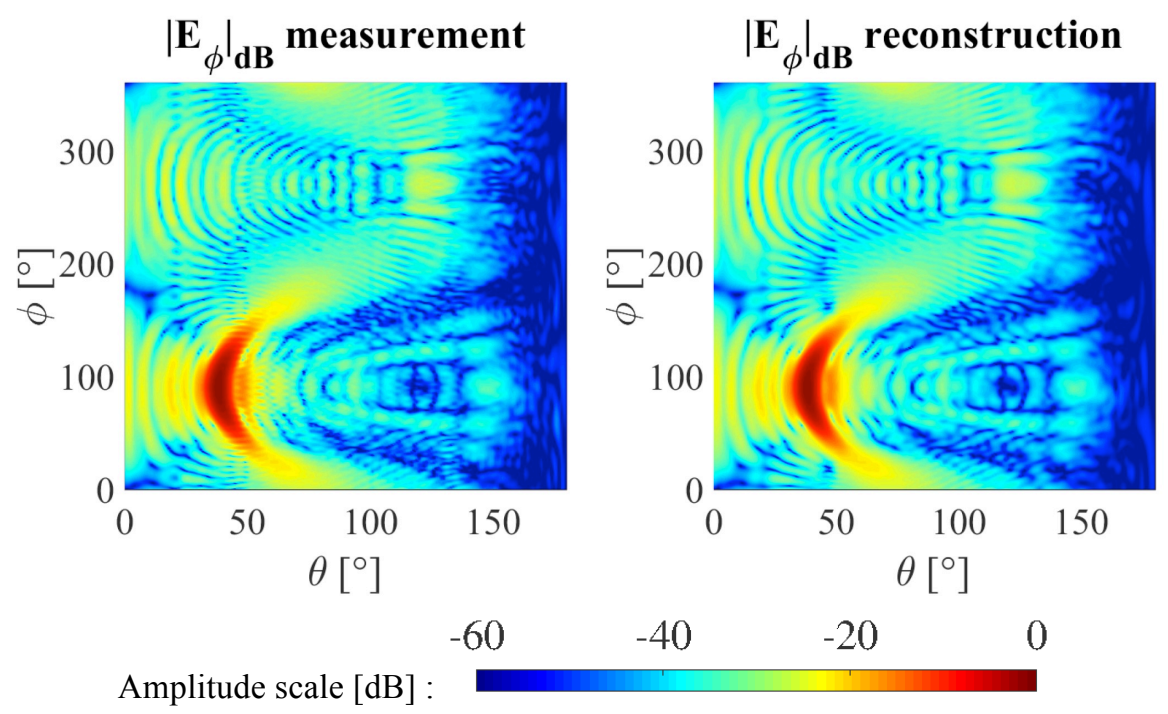



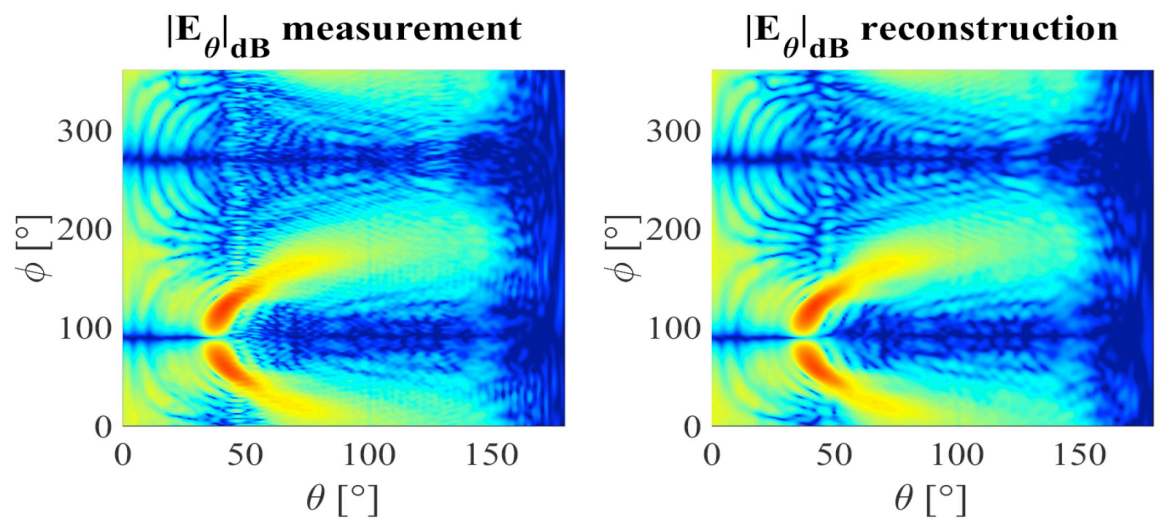

Figure 1. Far $E_{\theta}$ and $E_{\varphi}$ field 2D map in amplitude comparison of both the measured electric field and the reconstructed one for a flat metallic Luneburg lens antenna designed at $12 \mathrm{GHz}$ in the frame of MERLIN, a joint laboratory of Thalès Alenia Space and IETR.

1. C.D. Diallo, E. Girard, H. Legay, R. Sauleau, and O. Quevedo-Teruel, ”All-metal Ku-band Luneburg lens antenna based on variable parallel plate spacing Fakir bed of nails," published in 11th European Conference on Antenna and Propagation - EuCAP, March 2017, pp. 1401-1404. 